\title{
Apófise supracondilar do úmero: Causa rara de compressão alta do nervo mediano*
}

\section{Supracondylar Apophysis of the Humerus: Rare Cause of High Compression of the Median Nerve}

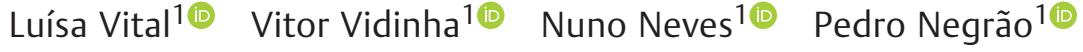 \\ ${ }^{1}$ Departamernto de Ortopedia e Traumatologia, Centro Hospitalar
Universitário de São João, Porto, Portugal \\ Rev Bras Ortop

\begin{abstract}
Endereço para correspondência Luísa Vital, MD, Departamento de Ortopedia e Traumatologia, Centro Hospitalar Universitário de São João, Alameda Professor Hernâni Monteiro, Porto, 4200 -319, Portugal (e-mail: luisavital1203@gmail.com).
\end{abstract}

\section{Resumo \\ Palavras-chave \\ - apófise supracondilar \\ - nervo mediano \\ - neuropatia compressiva \\ - compressão do nervo mediano}

A apófise supracondilar (ASC) é uma proeminência óssea que tem origem na face anteromedial do úmero distal com projeção inferior e que, apesar de habitualmente assintomática, pela relação com as estruturas adjacentes pode causar sintomatologia. Descrevemos o caso de uma mulher de 42 anos, com queixas álgicas irradiadas do cotovelo à mão, com 6 meses de evolução. Ao exame objetivo, a paciente apresentava um déficit sensorial no território do nervo mediano e diminuição da força de preensão. Foram realizadas radiografias do úmero distal nas quais era visível uma espícula óssea, e na ressonância magnética era evidente o espessamento do epineuro do nervo mediano. A eletromiografia apresentou uma desmielinização axonal grave do nervo mediano proximal ao cotovelo. Foi diagnosticada uma compressão do nervo mediano por uma ASC. A paciente foi submetida à cirurgia e 1 ano pós-operatório apresentou recuperação clínica total. A ASC é uma causa rara, mas possível e tratável da compressão alta do nervo mediano.

Supracondylar apophysis (SA) is a bony prominence that originates from the anteromedial aspect of the distal humerus with a lower projection and which, although usually asymptomatic, due to the relationship with adjacent structures can cause symptoms. We describe the case of a 42-year-old woman with pain complaints radiating from her elbow to her hand, with 6 months of evolution. On objective examination, the patient had a sensory deficit in the median nerve territory and decreased grip strength. Radiographs of the distal humerus were performed, in which a bone spike was visible, and magnetic resonance imaging showed thickening of the median nerve epineurium. Electromyography showed severe axonal demyelination of the median nerve proximal to the elbow. A median nerve compression caused by a SA was diagnosed. The patient underwent surgery and, 1 year after the operation, she had a complete clinical recovery. Supracondylar apolysis is a rare, but possible and treatable cause of high median nerve compression.
Trabalho desenvolvido no Departamento de Ortopedia e Traumatologia do Centro Hospitalar Universitário de São João, Porto, Portugal. recebido

29 de Abril de 2020

aceito

06 de Julho de 2020
DOI https://doi.org/

10.1055/s-0040-1718509. ISSN 0102-3616.
Copyright $\odot$ by Sociedade Brasileira de Ortopedia e Traumatologia. Published by Thieme Revinter Publicações Ltda, Rio de Janeiro, Brazil
License terms

(c) $9 \ominus \$$ 


\section{Introdução}

A apófise supracondilar (ASC)é uma estrutura anatômica descrita por Knox em 1841. Esta proeminência óssea de tamanho variável tem origem na face anteromedial do úmero distal, projeta-se inferiormente e representa um remanescente embriológico vestigial típico de animais trepadores. A banda fibrosa conhecida como ligamento de Struthers surge habitualmente como continuação da ASC e forma um forâmen no qual o nervo mediano e a artéria braquial podem ser comprimidos no seu trajeto. ${ }^{1}$ A prevalência desta estrutura anatômica varia amplamente nos estudos (0.7-2,5\%), sendo unânime, no entanto, que esta é rara e mais prevalente na raça caucasiana e no sexo feminino. ${ }^{2}$

\section{Relato de Caso}

Um paciente do sexo feminino, com 42 anos de idade, caucasiana, sem antecedentes médicos relevantes, foi observada em consulta de ortopedia por queixas álgicas progressivas, irradiadas do cotovelo até a mão esquerda, com 6 meses de evolução. Ao exame objetivo, a paciente apresentava um déficit sensorial no território do nervo mediano e diminuição da força de preensão. A paciente não apresentava tumefações

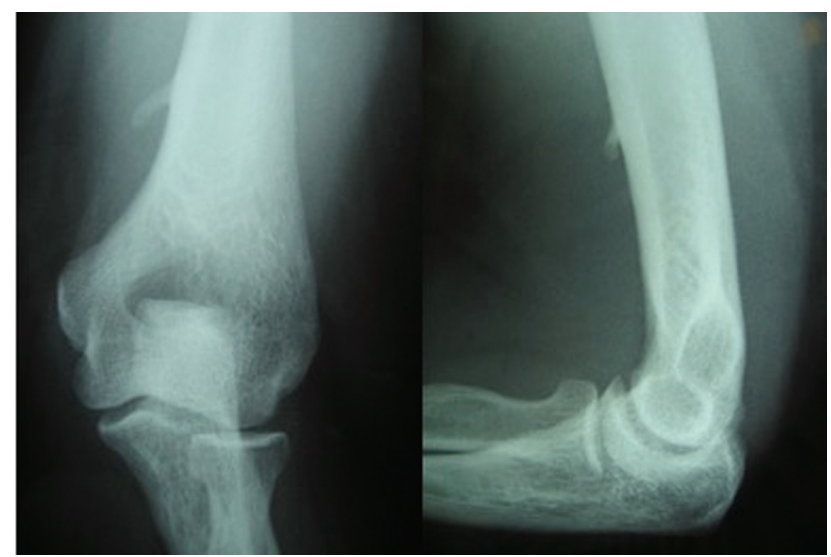

Fig. 1 Radiografias (incidência de face e perfil) do cotovelo esquerdo onde se observa a apófise supracondilar do úmero distal. palpáveis no membro superior esquerdo, nem Tinel positivo em todo o trajeto do nervo mediano ou alterações vasculares. Foi então submetida a exames de imagem para estudo da sintomatologia apresentada, que revelaram: nas radiografias do úmero distal, uma espicula óssea de orientação inferior ( - Figura 1) e na ressonância magnética, era evidente espessamento do epineuro do nervo mediano sugestivo de compressão nervosa. Na eletromiografia, a paciente apresentou uma desmielinização axonal grave do nervo mediano proximal ao cotovelo. Foi, desta forma, diagnosticada uma compressão do nervo mediano por uma ASC.

A paciente foi submetida à cirurgia para excisão desta estrutura por uma via anterior do úmero distal. Intraoperatoriamente confirmou-se a compressão do nervo mediano (-Figuras 2 e 3) e foi realizada a excisão da ASC e do ligamento de Struthers, sem intercorrências. Na avaliação pós-operatória aos 2 meses, a paciente apresentou uma melhoria significativa dos sintomas neurológicos com ganho na força de preensão e diminuição de parestesias. Foi realizada uma eletromiografia que mostrou uma recuperação apreciável do nervo mediano esquerdo com normalização da neurografia motora. Um ano após a cirurgia, houve uma regressão completa da sintomatologia e recuperação da força, equiparável ao lado contralateral.

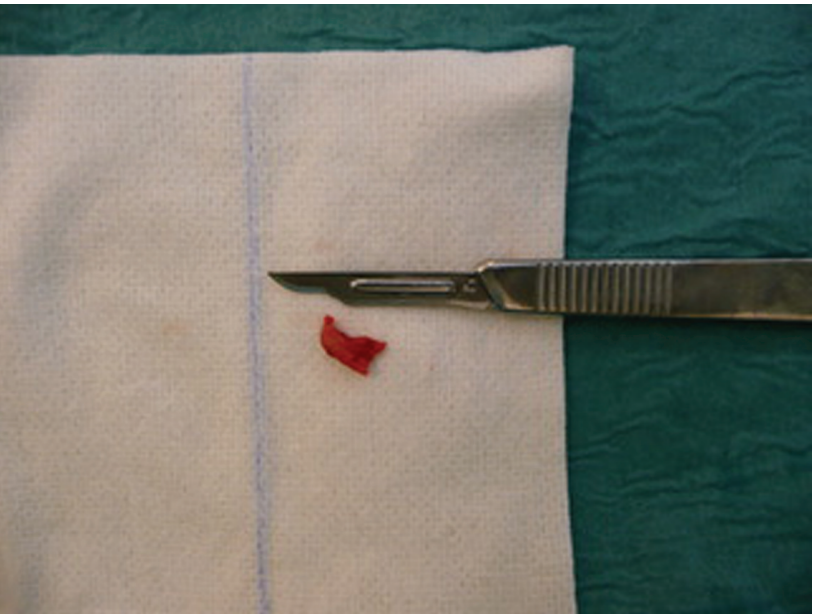

Fig. 3 Imagem da apófise supracondilar após a sua excisão.

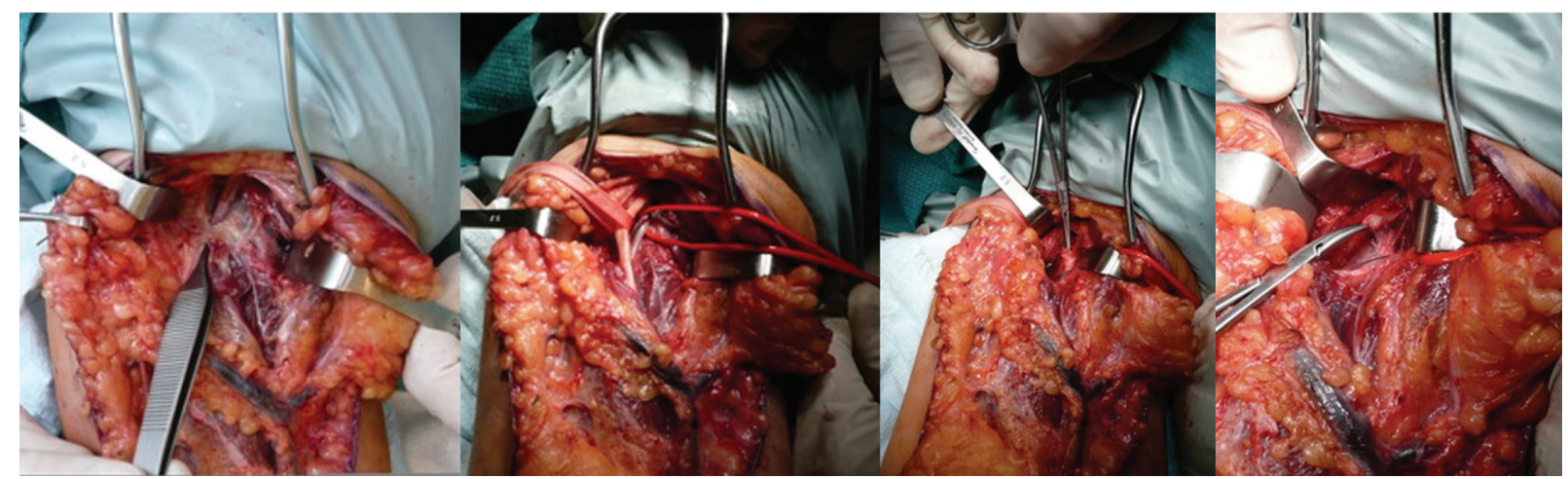

Fig. 2 Imagens intraoperatórias da dissecção anterior por planos. Observação de várias estruturas envolvidas: nervo mediano, artéria braquial, ligamento de Struthers e apófise supracondilar. 


\section{Discussão}

A ASC é uma estrutura anatômica habitualmente sem manifestação clínica; no entanto, em alguns casos, pode se tornar sintomática e manifestar-se por tumefação e/ou sintomas de compressão do nervo mediano e da artéria braquial. ${ }^{3}$ Soliere ${ }^{4}$ relatou, em 1929, o primeiro caso de alterações clínicas causadas pela presença de uma ASC. Esta entidade representa um desafio diagnóstico dada a apresentação clínica semelhante à neuropatia mais comum do membro superior Síndrome do túnel do carpo e também pelos múltiplos locais possíveis de compressão do nervo mediano ao nível do cotovelo, que incluem: entre as cabeças do pronador redondo, na arcada aponeurótica formada pela inserção proximal dos músculos flexores do antebraço, e no lacertus fibrosus. ${ }^{5}$ Também deve fazer parte dos diagnósticos diferenciais a possibilidade de se tratar de ossificação heterotópica ou osteocondroma, sendo que este apresenta características diferenciadoras que passam pela orientação da espicula óssea-não apontando em direção à articulação e continuando com a cortical do úmero. ${ }^{6}$

Alguns casos clínicos de compressão neurovascular associados a esta estrutura estão descritos na literatura: Aydinlioglu et al. ${ }^{7}$ descreveram um caso de compressão bilateral do nervo mediano pela ASC; May-Miller et al. ${ }^{8}$ reportaram um caso raríssimo de compressão do nervo cubital, e existem também relatos de fratura desta estrutura. ${ }^{9}$

Na suspeita clínica de neuropatia pela ASC, os exames de imagem aliados à eletromiografia são diagnósticos, como no caso clínico que descrevemos. O tratamento preconizado em doentes sintomáticos é cirúrgico e consiste na exérese da ASC e, caso presente, do ligamento de Struthers, permitindo desta forma confirmar a descompressão das estruturas envolvidas. Tal como descrito na literatura e verificado neste caso clínico, esta opção de tratamento está associada a bons resultados funcionais a curto e longo prazo. ${ }^{10}$

Concluindo, a ASC é uma causa rara, mas possível e tratável da compressão alta do nervo mediano.

\section{Conflito de Interesses}

Os autores declaram não haver conflito de interesses.

\section{Referências}

1 Kessel L, Rang M. Supracondylar spur of the humerus. J Bone Joint Surg Br 1966;48(04):765-769

2 C S, B S S, G v K, S L. Morphological study of the supracondylar process of the humerus and its clinical implications. J Clin Diagn Res 2014;8(01):1-3 [REMOVED HYPERLINK FIELD]

3 Subasi M, Kesemenli C, Necmioglu S, Kapukaya A, Demirtas M. Supracondylar process of the humerus. Acta Orthop Belg 2002;68 (01):72-75

4 Solieri S. Nervalgia del nervo mediano da processo sopraepitrocleare. Chir Organi Mov 1929;14:171-175

5 Caetano EB, Sabongi JJ, Vieira LA, Caetano MF, de Bona JE, Simonatto TM. Struthers' ligament and supracondylar humeral process: an anatomical study and clinical implications. Acta Ortop Bras 2017;25(04):137-142

6 Fragiadakis EG, Lamb DW. An unusual cause of ulnar nerve compression. Hand 1970;2(01):14-16

7 Aydinlioglu A, Cirak B, Akpinar F, Tosun N, Dogan A. Bilateral median nerve compression at the level of Struthers' ligament. Case report. J Neurosurg 2000;92(04):693-696

8 May-Miller P, Robinson S, Sharma P, Shahane S. The Supracondylar Process: A Rare Case of Ulnar Nerve Entrapment and Literature Review. J Hand Microsurg 2019;11(Suppl 1):S06-S10

9 Newman A. The supracondylar process and its fracture. Am J Roentgenol Radium Ther Nucl Med 1969;105(04):844-849

10 Shon HC, Park JK, Kim DS, Kang SW, Kim KJ, Hong SH. Supracondylar process syndrome: two cases of median nerve neuropathy due to compression by the ligament of Struthers. J Pain Res 2018; $11: 803-807$ 